\title{
Author Index Vol 46, 1983
}

$\mathrm{A}=$ Abstracts

Afshar, F. 147 Allen, A.H. 26 Allen, CD. 26 Amano, K. 311(A) Andermann, F. 33 Andy, OJ. 62,107,116

Baba, H. 308 (A) Bedenbaugh, P. 160 Beique, R.A. 52,227 Bertrand, G. 37, 84, 200 ,

272 Bouvier, G. 52,227 Braun.D.P. 240 Broggi, G. 236 Bullard,D.E. 188 Buren, G. 206 Burger, P.C 180,188 Byrd, R. 188

Cappabianca, P. 295 Caputi.F. 295 Cerchiari, U. 236 Chiu,K.M. 240 Comte, P. 41 Corrie.W.S. 47 Cosman, E.R. 160 Couillard, P. 227

Dafny.N. 254 Davis,R. 57

Del Basso De Caro, M. 295 Denda,Y. 317 (A) Dieckmann, G. 206 Dimitrijevic, M.R. 124,

245 Divitiis, E. de 295 Dolenc.V. 124 
Dubois, P. 188 Dusnak, A. 57 Dykes, E. 147

Eguchi,T. 313 (A) Engle, H. 57

Faganel, J. 245 Ferguson, S.M. 47 Fiume, D. 290 Florea-Ciocoiu, V. 261 Franzini, A. 236 Friedman, A. 188 Fröder, M. 206 Fujimoto.N. 307 (A) Fukami,T. 317 (A) Funahashi, K. 310 (A)

Garibotto.G. 236 Giard, N. 52 Gildenberg, P.L. 170 Ginsberg, M. 211 Giorgi,C 236 Gloor, P. 33 Goerss,S. 193 Gorkisch.K. 286 Gray, E. 57

Halter, J. 124 Hanamura, T. 313 (A) Hardy.T.L. 217 Harris.J.E. 240

Hawrylyshyn, P. 68 Hilaire, J.M.S. 52 Hirai, T. 317 (A), 318 (A) Hirato, M. 317

(A), 318 (A) Hondo,H. 307 (A) Hood.T.W. 19 
Hosobuchi, Y. 112 Houdek, P. 211

Iai.S. 313 (A) Ikeda, S. 307 (A) Imamura, Y. 315 (A) Iseki, H. 311 (A) Ishijima, B. 310 (A) Ito.T. 309 (A) Iwabuchi.T. 315 (A) Iwata, K. 308 (A)

Jurko, M. 62

Kageyama, N. 308 (A) Kakinoki, Y. 311 (A) Kali, B. 193 Kaminogo, M. 308 (A)

Katakura, R. 307 (A) Katayama, Y. 312 (A) Kawabatake, H. 311 (A) Kawamura, H. 311 (A) Kawashima, Y. 317 (A),

318 (A) Kayama,T. 307 (A) Kelly, P.J. 167,193 Kitamura, K. 310 (A),

311 (A), 316 (A) Knierim, D. 231 Koch,J. 217 Komai, N. 310 (A) Koslow.M. 236

Kroin.J.S. 240 Kuwahara, K. 307 (A)

Labrecque, R. 52 Lassiter, A. 217 Law.J.D. 129 


\section{Author Index}


Leger, S.L. 227 Lesage, J. 227

Maeda,T. 313 (A) Manaka,S. 315 (A) Marcussen, W.H. 276 Mathumoto, Y. 314 (A) Matsumoto.K. 307 (A),

317 (A) Mayanagi, Y. 313 (A) Mercier, C. 52 Miyamoto, E. 310 (A) Miyazaki.M. 318 (A) Miyazaki.S. 312 (A) Mori, K. 308 (A) Murayama,Y. 307 (A),

$317 \quad$ (A)

Nagaseki.Y. 317 (A),

318 (A) Nakagaki,H. 316 (A) Nakajima,S. 309 (A) Nakao.Y. 314 (A) Nakaoka.T.

92, 315 (A) Namba,S. 314 (A) Narabayashi, H. 313 (A),

319 (A) Nashold, B.S. 160,188 Nishlmoto, A. 314 (A) Nishimoto, H. 312 (A)

Nishimura, S. 308 (A) Nizuma,H. 307 (A) Notani, M. 311 (A)

Oakes.W.J. 188 Ohmoto.T. 314 (A) Ohyama, H. 307 (A) Ohye, C. 313 (A), 317 (A), 318 (A) Ojemann, G.A. 11 Olivier, A. 33,37,84,200,

272 Ono, K. 308 (A) 
Organ,L.W. 68 Osborne, D. 188 Otsuki.T. 307 (A)

Pagura.J.R. 138,154 Passarelli, P. 154 Penn, R.D. 240 Peters, T. 37 Peters, T.M. 200 Pettinato, G. 295 Picard,C. 84

Quesney, L.F. 33

Rayport, M. 47 Reeves, A.G. 26 Remler, M.P. 276 Reyes-Vazquez, C. 254 Ritland, S. 231 Roberts, D.W. 26 Rogozea, R. 261 Rotent,F. 227 Ryu, H. 309 (A)

Sakamoto, M. 317 (A) Sano, K. 315 (A) Schnapp, M. 154 Schold, C. 188 Seitzer, D. 206 Sekino, H. 315 (A) Sharkey, P.C. 124 Sherwood, A.M. 245 Shibazaki.T. 317 (A), 318 (A) Shibuya, H. 312 (A) Shichijo, F. 317 (A) Shima, F. 316 (A) Shimizu, H.

310(A) Shiwaku.T. 311 (A) Shozuhara, H. 309 (A) Siegfried, J. 19,41 Siqueira. J. 68 Soga.T. 307 (A) 
Sogabe, K. 307 (A), 317 (A) Spaziante, R. 295 Spiegel, E.A. 7,320 Stahl.H. 286

Straschill, M. 286 Sukurai, M. 314 (A) Suzuki, I. 313 (A) Suzuki, J. 307 (A)

Takahashi.H. 310 (A) Takahashi.T. 315 (A) Takakura, K. 313 (A) Tanikawa, T. 311 (A) Tari.A. 313 (A) Tasker, R.R. 68, 311 (A) Tosaki, F. 308 (A) Trioio, P. 188

Tsubokawa, T. 312 (A) Tsuda, T. 311 (A), 317 (A) Tsukamoto, Y. 315 (A) Tsukiyama, T. $312(\mathrm{~A})$

Uemura, K. 309 (A)

Van Buren, J.M. 211

Wada, H. 317 (A), 318 (A) Watanabe, E. 313 (A) Watanabe, S. 308 (A) Watkins. E.S. 147 Wieser.H.G. 19,41

Yabumoto, M. 310(A) Yamada.S. 231 Yamamoto, H. 308 (A) Yamamoto, T. 312 (A) Yamazaki.A. 308 (A) Yasue, M. 310 (A) Yokochi, F. 313 (A) Yoshioka, M. 315 (A) Yuasa, H. 308 (A)

Zukic,A. 167 